\title{
Existence of Forbidden Digraphs for Crisp Boolean Petri Nets
}

\author{
Gajendra Pratap Singh \\ School of Computational and Integrative Sciences, \\ Jawaharlal Nehru University, New Delhi-110067, India. \\ Corresponding author: gajendra@mail.jnu.ac.in \\ Sujit Kumar Singh \\ School of Computational and Integrative Sciences, \\ Jawaharlal Nehru University, New Delhi-110067, India. \\ E-mail: sujit36_sit@jnu.ac.in \\ Madhuri Jha \\ School of Computational and Integrative Sciences, \\ Jawaharlal Nehru University, New Delhi-110067, India. \\ E-mail: jhamadhuri81@gmail.com
}

(Received March 15, 2019; Accepted September 8, 2019)

\begin{abstract}
Boolean Petri net (BPN) and Crisp Boolean Petri net (CBPN) is a well-studied graph model since 2010 which has several applications in mathematical modeling of complex or tricky networks. Modeling any network with Petri net which can generate binary numbers as marking vectors in its reachability tree is still has much uses. In CBPN with a minimum number of transition and minimum number of steps of reachability tree, minimal execution time to run the machine has not been noted till date, thus it's necessary to sort out this problem. Possibly it may occur due to some forbidden structure which hinders any 1-safe Petri net to be a CBPN. In this paper, we present some forbidden digraphs whose presence interrupts the generation of binary $n$-vectors exactly once. Any 1 -safe Petri net is not a CBPN if it contains any of the subnet induced to the four forbidden structures discussed in this paper.
\end{abstract}

Keywords- Petri net, Binary vectors, Boolean Petri net, Crisp Boolean Petri net, Forbidden graphs.

\section{Introduction}

In the field of modeling any real-world system, Petri net is one of the emerging theories from the early '90s. Due to its versatile modeling tools, it is easy to model any type of system preserving the basic properties of the system (Murata, 1989). It is a mathematical and graphical approach to design and study the behavior of the system. Petri net modeling provides graphical appealing language which is appropriate for modeling system having conflict or concurrency (Jenson, 1993). It is also used to describe and analyze the complex problems rather than the method of flow chart. There are several types of Petri net to deal with a different system like Boolean Petri nets, Colored Petri net, Fuzzy Petri net, Timed Petri net etc. (Kansal et al., 2010, 2012 and 2015; Jenson and Rozenberg, 2012; Rosen and Krithivasan, 2012; Gupta et al., 2019a). Previously in (Kansal et al., 2011b), characterization of a specific class in Petri nets is being discussed which are known as Boolean Petri net (BPN). Boolean Petri net is further classified as Crisp Boolean Petri net (CBPN) by applying suitable conditions (Kansal et al., 2011a). After classification, a certified algorithm is always required for the characterization of any special type of graph and that should be not in polynomial time but in linear time. Certainly, there exist some graphs like Planer graph and Trees which has the certifying algorithm for the characterization (Harary, 2015). Similar arguments are needed for 
International Journal of Mathematical, Engineering and Management Sciences

Vol. 5, No. 1, 83-95, 2020

https://doi.org/10.33889/IJMEMS.2020.5.1.008

BPN or CBPN, but the situation is slightly different in this case. When for any Petri net if the possible number of reachable states does not follow a linear time algorithm, then it may occur due to the existence of some forbidden structures in the model. Boolean refers to binary where Binary System is very much useful to study as it has several applications in every field of sciences. It is used in computers and other electronic devices which deal with two symbols only ' 0 ' and ' 1 ' while the decimal number system deals with 10 symbols. The two-digit used here represents two states (ON/OFF) and these states used to carry the instruction and to store data. It is useful to find the system where all the binary vectors are generated and more precisely to generate the vectors without repetition, e.g., in gene expression, cell division, decision science. So, it is useful to study the forbidden digraph for the system which does not allow to generate all the binary vectors. Petri net theory can be used to describe lots of important families of networks with a finite set of isolated nets which does not include into the family and in addition to that one can avoid including all these structures from that family which contain any of these forbidden digraphs as (induced) subgraph or subnet. By saying Crisp Boolean Petri nets, it means that a 1-safe Petri net that produces each binary $n$-vector only once in its reachability graph. In this paper, we propose the structure of some forbidden subnets which hinders any 1-safe Petri net to be a CBPN. These forbidden digraphs interrupt in the generation of all the binary $n$-vectors exactly once in its reachability tree as its marking vector (Pastor et al., 1994; Kansal et al., 2012). Petri net modeling has other applications too, like it can behave as a recommender system or can be modeled for many biological networks which can be proven very helpful to study the complex biological networks or disease networks (Kumar et al., 2018; Gupta et al., 2019b; Singh and Gupta, 2019; Singh and Singh, 2019).

\section{Some Preliminary Definitions}

\subsection{Petri Nets}

Petri nets are the special types of the directed bipartite graph which has two kinds of nodes, i.e., places and transition that are connected by directed edge or arcs. Petri nets have been proven useful for dynamical modeling, analysis, and verifying protocols especially using in networks. This came in focus when Carl Adam Petri used this theory in his thesis in 1962 and by then PN being used to model the system in almost every field of science increasingly (Petri and Reisig, 2008). Several scientists define Petri net in their own way according to their system but with the same basic structure of Petri net. A Petri net is composed of mainly five components represented mathematically as:

$$
P N=\left(P, T, I^{-}, I^{+}, \mu^{0}\right)
$$

where,

- $\quad P$ is a nonempty finite set of places,

- $T$ is a nonempty finite set of transitions,

- $I^{-}$is the incidence function from places to transition,

- $I^{+}$is the incidence function from transition to place,

- $\mu^{0}$ is the initial marking, denoted by dots, called tokens.

Graphically, in Petri net, transitions are drawn as rectangles and places as circles or ovals and arrow used to represent flow relation. A transition $t \in T$ is said to be ready to fire at the marking $\mu^{0}$ if and only if the number of directed arcs from places to transitions is less than equal to the number of tokens in its input place $\forall p \in P$. After firing at $\mu^{0}$ the new marking $\mu^{1}$ is given by the rule, 
International Journal of Mathematical, Engineering and Management Sciences

Vol. 5, No. 1, 83-95, 2020

https://doi.org/10.33889/IJMEMS.2020.5.1.008

$$
\mu^{1}(p)=\mu^{0}(p)-I^{-}(p, t)+I^{+}(p, t), \quad \forall p \in P .
$$

If any transition $t$ fires at $\mu^{0}$ to yield $\mu^{1}$, then $\mu^{1}$ is said to be reachable directly from $\mu^{0}$. There must exist a firing sequence of transitions to obtain $\mu^{1}$ from $\mu^{0}$. The set of all reachable markings of a $P N$ along with the arcs is called the reachability graph, denoted by $R\left(P N, \mu^{0}\right)$. Algorithm for reachability problem is described in (Mayr, 1984). An example of Petri net and a firing sequence is shown in Figure 1.

An illustration for Petri net dynamics:

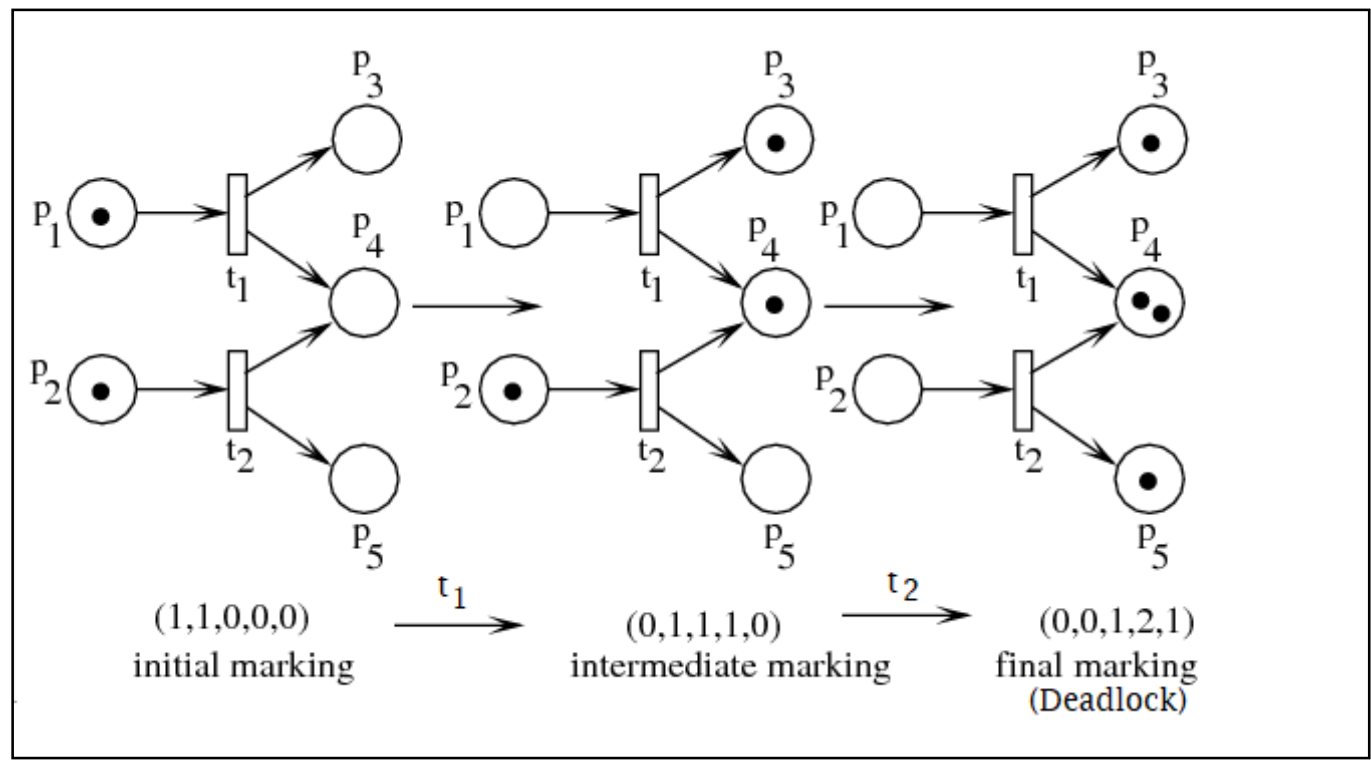

Figure 1. Petri net and one firing sequence

Any transition is said to be sink if it does not have any output place and a transition is said to be a source if it does not have any input place. A sink transition always consumes the token from its input place and source transition always produce tokens to its output places. A transition is said to be dead transition if it never fires.

\subsection{1-Safe Petri Net}

A Petri net is called 1-safe Petri net if the number of tokens at any place can never exceed one. It can also be referred to as a special case of $k$-bounded Petri net, by putting $k=1$.

\subsection{Boolean Petri Net}

In mathematics, binary number system means 2 as the base and it requires only two different symbols ' 0 ' and ' 1 ', different from the usual ' 10 ' different symbols required in the decimal system (Piestrak, 1995). A Petri net is a Boolean Petri net if it generates all the binary vectors in its reachability graph (Kansal et al., 2011b) (see Figure 2). 


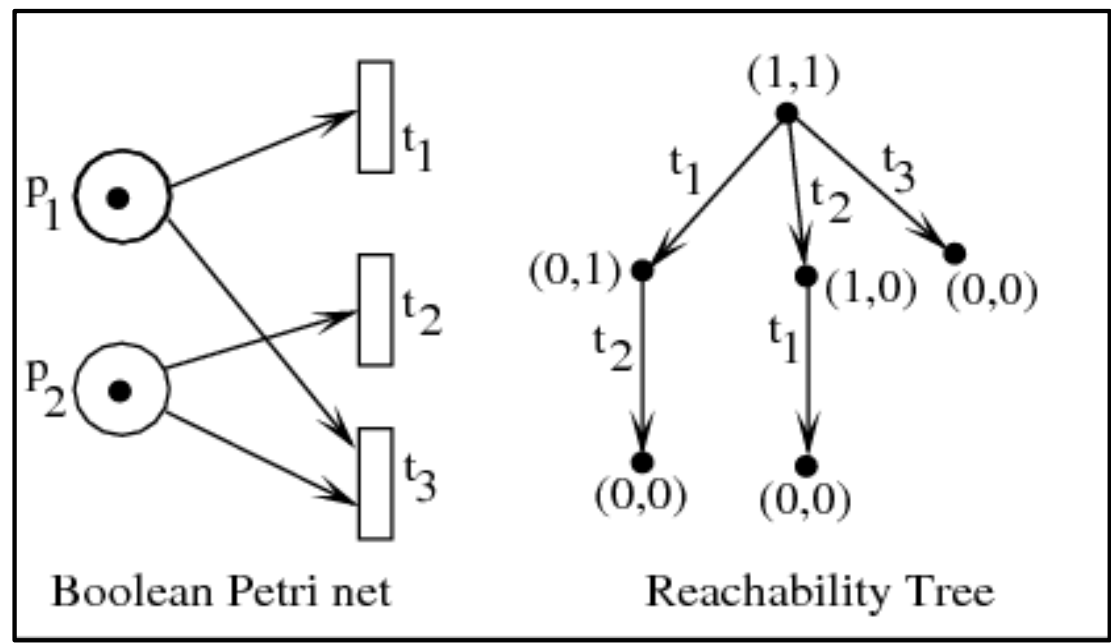

Figure 2. Boolean Petri net and its reachability tree

\subsection{Crisp Boolean Petri Net (CBPN)}

A Petri net is Crisp Boolean Petri net if it generates all the binary vectors exactly once in its reachability graph (see Figure. 3). Earlier we have discussed the results on Crisp Boolean Petri net and its construction from a 1-safe Petri net (Kansal et al., 2011a).

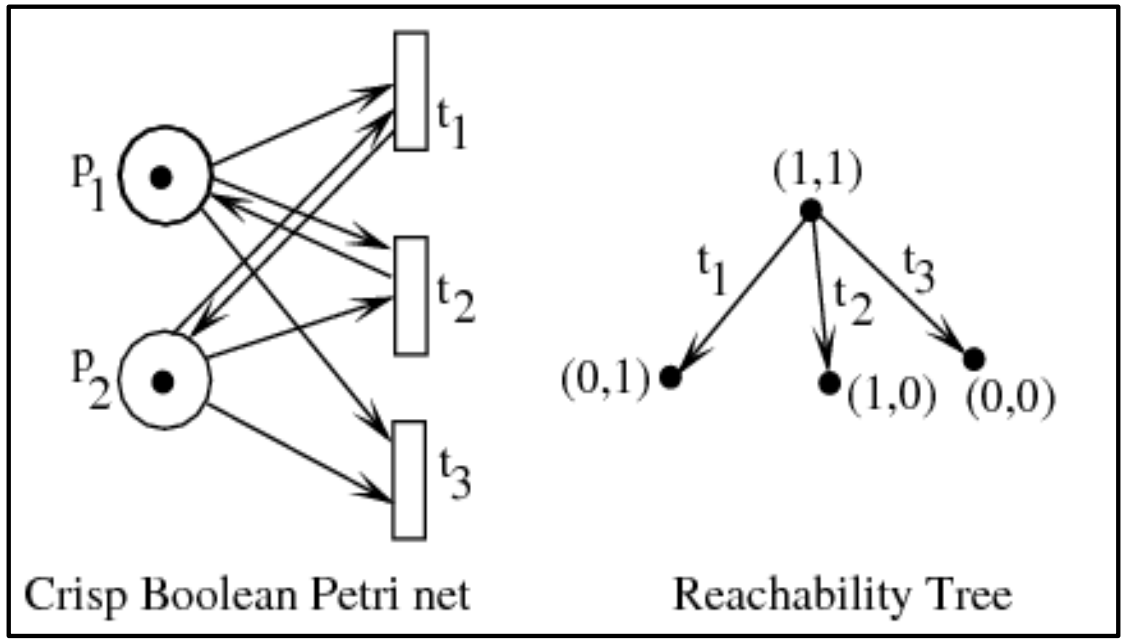

Figure 3. Crisp boolean Petri net and its reachability graph

\subsection{Forbidden Digraphs}

Forbidden Digraphs are defined as a finite set of individual graphs in which each member does not belong to the original family and further excluded from that family which contains any one of these forbidden graphs as (induced) subgraphs or its homeomorphic images. For example, a tree can be defined as a connected graph that does not contain cycles, thus cycles are forbidden for being a tree. 
International Journal of Mathematical, Engineering and Management Sciences

Vol. 5, No. 1, 83-95, 2020

https://doi.org/10.33889/IJMEMS.2020.5.1.008

Planar graphs are the graphs which can be embedded in a plane while talking about its forbidden structures one can say that graphs should not contain induced subgraphs from the complete $K_{5}$ graph or the complete bipartite graph $K_{3,3}$. In (Francis et al., 2015) authors characterized some forbidden structures for Circular-arc graphs.

\section{Result on Forbidden Digraphs for Crisp Boolean Petri net}

Statement: For any Crisp Boolean Petri net (CBPN), there exist four forbidden smallest subnets which hinder 1-safe Petri net to be a CBPN.

or

Any 1-safe Petri net is not Crisp Boolean Petri net if it contains any of the four forbidden structures shown in Figure 4 as its induced subnet.

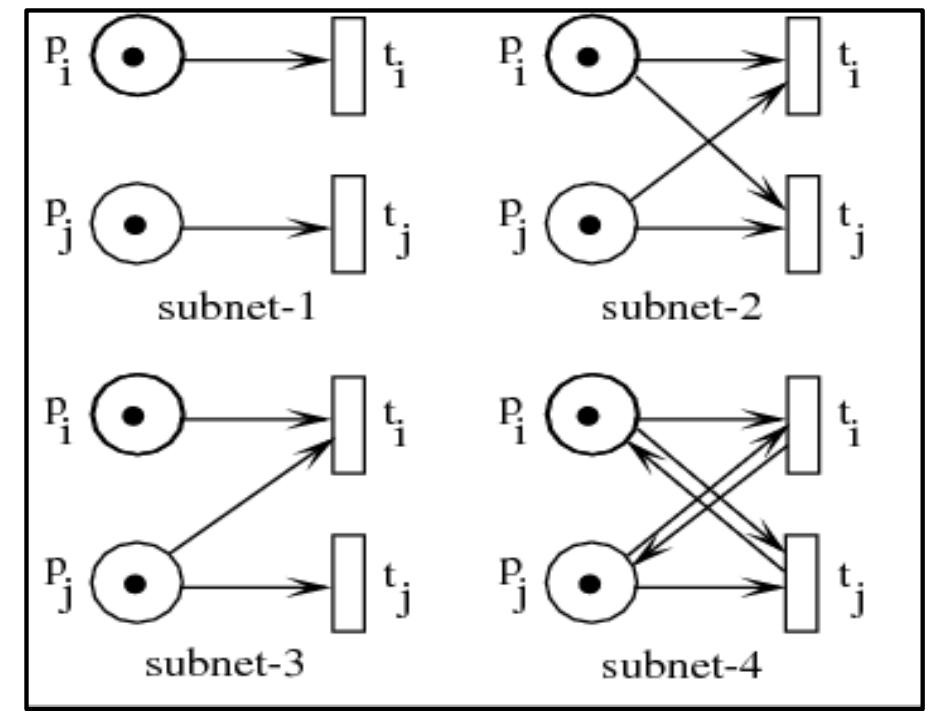

Figure 4. Forbidden structures

Proof: Let $P N=\left(P, T, I^{-}, I^{+}, \mu^{0}\right)$ a 1-safe Petri net that means the upper bound of the number of tokens on any place is one. Let us suppose the place set $P$ contains $n$ number of places i.e., $P=\left\{p_{1}, p_{2}, \ldots, p_{i}, p_{j}, \ldots, p_{n}\right\}$ and transition set contains $m$ number of transitions i.e., $T=$ $\left\{t_{1}, t_{2}, \ldots, t_{i}, t_{j}, \ldots, t_{m}\right\}$.

In the generation of binary $n$-vectors exactly once, i.e., to be a CBPN, 1 -safe PN should not contain the four above subnet structures as its induced subnet. We will prove this result by the method of contradiction. For this let us suppose the given PN is a CBPN and contains any one of the forbidden structures and the contractions obtained in the generation of binary $n$-vectors is shown separately for each subnet.

\subsection{For Subnet-1}

Let us suppose PN contains subnet- 1 as an induced subnet. Let us consider $\mu^{0}\left(p_{k}\right)=1, \forall p_{k} \in P$ in PN. If we consider only the subnet-1 then according to the firing rule $t_{i}$ will fire either separately or simultaneously. Here, we are taking the case of firing separately. 
International Journal of Mathematical, Engineering and Management Sciences

Vol. 5, No. 1, 83-95, 2020

https://doi.org/10.33889/IJMEMS.2020.5.1.008

As,

$$
\begin{gathered}
I^{-}\left(p_{i}, t_{i}\right) \leq \mu^{0}\left(p_{i}\right) \\
1 \leq 1 .
\end{gathered}
$$

This makes $t_{i}$ fires and hence the next marking will be changed only for $p_{i}$ as $p_{j}$ is not connected with $t_{i}$. The next marking will be by the rule

and thus $\mu_{t_{i}}^{1}=(1,1, \ldots, 0,1, \ldots, 1)$.

$$
\begin{aligned}
\mu_{t_{i}}^{1}\left(p_{i}\right) & =\mu^{0}\left(p_{i}\right)-I^{-}\left(p_{i}, t_{i}\right)+I^{+}\left(p_{i}, t_{i}\right) \\
& =1-1+0=0
\end{aligned}
$$

$$
\text { Again, } \begin{aligned}
I^{-}\left(p_{j}, t_{j}\right) & \leq \mu^{0}\left(p_{j}\right) \\
1 & \leq 1 .
\end{aligned}
$$

This making $t_{j}$ fires and hence the next marking will be changed only for $p_{j}$ as $p_{i}$ is not connected with $t_{j}$.

$$
\begin{aligned}
\mu_{t_{j}}^{1}\left(p_{j}\right) & =\mu^{0}\left(p_{j}\right)-I^{-}\left(p_{j}, t_{j}\right)+I^{+}\left(p_{j}, t_{j}\right) \\
& =1-1+0=0 .
\end{aligned}
$$

Thus, $\quad \mu_{t_{j}}^{1}=(1,1, \ldots, 1,0, \ldots, 1)$.

Now, with $\mu_{t_{i}}^{1}=(1,1, \ldots, 0,1, \ldots, 1)$ only $t_{j}$ satisfy the enabling condition

$$
\begin{aligned}
I^{-}\left(p_{j}, t_{j}\right) & \leq \mu_{t_{j}}^{1}\left(p_{j}\right) \\
1 & \leq 1 .
\end{aligned}
$$

Thus, $t_{j}$ will fire to change the marking of $p_{j}$ only. The new marking will be:

$$
\begin{aligned}
\mu_{t_{i}, t_{j}}^{2}\left(p_{j}\right) & =\mu_{t_{i}}^{1}\left(p_{j}\right)-I^{-}\left(p_{j}, t_{j}\right)+I^{+}\left(p_{j}, t_{j}\right) \\
& =1-1+0=0 .
\end{aligned}
$$

Thus, the next marking will be: $\mu_{t_{i}, t_{j}}^{2}=(1,1, \ldots, 0,0, \ldots, 1)$.

Again by taking $\mu_{t_{j}}^{1}=(1,1, \ldots, 1,0, \ldots, 1)$, only $t_{i}$ fires as

$$
\begin{aligned}
I^{-}\left(p_{i}, t_{i}\right) & \leq \mu_{t_{j}}^{1}\left(p_{i}\right) \\
1 & \leq 1 .
\end{aligned}
$$

Thus, $t_{i}$ will fire to change the marking of $p_{i}$ only. The new marking will be:

$$
\begin{aligned}
\mu_{t_{j}, t_{i}}^{2}\left(p_{i}\right) & =\mu_{t_{j}}^{1}\left(p_{i}\right)-I^{-}\left(p_{i}, t_{i}\right)+I^{+}\left(p_{i}, t_{i}\right) \\
& =1-1+0=0 .
\end{aligned}
$$

Thus, the next marking will be: $\mu_{t_{j}, t_{i}}^{2}=(1,1, \ldots, 0,0, \ldots, 1)$.

Here, the concept of crispness is contradicting as:

$$
\mu_{t_{i}, t_{j}}^{2}=\mu_{t_{j}, t_{i}}^{2}=(1,1, \ldots, 0,0, \ldots, 1) .
$$

Thus, if Petri net consisting of subnet- 1 then repetition of Binary $n$-vector occurs which contradicts 
International Journal of Mathematical, Engineering and Management Sciences

Vol. 5, No. 1, 83-95, 2020

https://doi.org/10.33889/IJMEMS.2020.5.1.008

the concept of Crispness. Hence, subnet-1 is the forbidden structure for crisp Boolean Petri net.

\subsection{For Subnet-2}

Let us suppose PN contains this subnet- 2 as an induced subnet, only by considering this subnet we can conclude the result for the whole Petri net. Here according to the firing rule both $t_{i}$ and $t_{j}$ are enabled either they fire simultaneously or separately. We will show the firing separately, as

$$
\begin{gathered}
I^{-}\left(p_{i}, t_{i}\right) \leq \mu^{0}\left(p_{i}\right)=>1 \leq 1 \\
I^{-}\left(p_{j}, t_{i}\right) \leq \mu^{0}\left(p_{j}\right)=>1 \leq 1
\end{gathered}
$$

Thus, $\mathrm{t}_{\mathrm{i}}$ will fire and hence the next marking will change for both $p_{i}$ and $p_{j}$.

and,

$$
\begin{aligned}
\mu_{t_{i}}^{1}\left(p_{i}\right) & =\mu^{0}\left(p_{i}\right)-I^{-}\left(p_{i}, t_{i}\right)+I^{+}\left(p_{i}, t_{i}\right) \\
& =1-1+0=0
\end{aligned}
$$

$$
\begin{aligned}
\mu_{t_{i}}^{1}\left(p_{j}\right) & =\mu^{0}\left(p_{j}\right)-I^{-}\left(p_{j}, t_{i}\right)+I^{+}\left(p_{j}, t_{i}\right) \\
& =1-1+0=0 .
\end{aligned}
$$

Thus,

$$
\mu_{t_{i}}^{1}=(1,1, \ldots, 0,0, \ldots, 1)
$$

Again,

$$
\begin{aligned}
& I^{-}\left(p_{i}, t_{j}\right) \leq \mu^{0}\left(p_{i}\right)=>1 \leq 1 \\
& I^{-}\left(p_{j}, t_{j}\right) \leq \mu^{0}\left(p_{j}\right)=>1 \leq 1
\end{aligned}
$$

This makes $t_{j}$ fires and hence the next marking will be changed for $p_{i}$ and $p_{j}$, as both are connected with $t_{j}$.

$$
\begin{aligned}
\mu_{t_{j}}^{1}\left(p_{i}\right) & =\mu^{0}\left(p_{i}\right)-I^{-}\left(p_{i}, t_{j}\right)+I^{+}\left(p_{i}, t_{j}\right) \\
& =1-1+0=0
\end{aligned}
$$

and,

$$
\begin{aligned}
\mu_{t_{i}}^{1}\left(p_{j}\right) & =\mu^{0}\left(p_{j}\right)-I^{-}\left(p_{j}, t_{j}\right)+I^{+}\left(p_{j}, t_{j}\right) \\
& =1-1+0=0 .
\end{aligned}
$$

Thus,

$$
\mu_{t_{j}}^{1}=(1,1, \ldots, 0,0, \ldots, 1)
$$

Here, this contradicts the concept of crispness

$$
\mu_{t_{i}}^{1}=\mu_{t_{j}}^{1}=(1,1, \ldots, 0,0, \ldots, 1) \text {. }
$$

\subsection{For Subnet-3}

Let us suppose PN contains subnet- 3 structure as an induced subnet. Then according to the firing rule $t_{i}$ and $t_{j}$ will fire either separately or simultaneously. Here, we are taking the case of firing separately. 
International Journal of Mathematical, Engineering and Management Sciences

Vol. 5, No. 1, 83-95, 2020

https://doi.org/10.33889/IJMEMS.2020.5.1.008

As,

$$
\begin{gathered}
I^{-}\left(p_{i}, t_{i}\right) \leq \mu^{0}\left(p_{i}\right)=>1 \leq 1 \\
I^{-}\left(p_{j}, t_{i}\right) \leq \mu^{0}\left(p_{j}\right)=>1 \leq 1
\end{gathered}
$$

This making $t_{i}$ fires and hence the next marking will be changed only for $p_{i}$ as well as for $p_{j}$ As both are connected with $t_{i}$. Thus, the new marking will be

$$
\begin{aligned}
\mu_{t_{i}}^{1}\left(p_{i}\right) & =\mu^{0}\left(p_{i}\right)-I^{-}\left(p_{i}, t_{i}\right)+I^{+}\left(p_{i}, t_{i}\right) \\
& =1-1+0=0
\end{aligned}
$$

and

$$
\begin{aligned}
\mu_{t_{i}}^{1}\left(p_{j}\right) & =\mu^{0}\left(p_{j}\right)-I^{-}\left(p_{j}, t_{i}\right)+I^{+}\left(p_{j}, t_{i}\right) \\
& =1-1+0=0 .
\end{aligned}
$$

Thus, $\mu_{t_{i}}^{1}=(1,1, \ldots, 0,0, \ldots, 1)$.

Again,

$$
\begin{aligned}
I^{-}\left(p_{j}, t_{j}\right) & \leq \mu^{0}\left(p_{j}\right) \\
1 & \leq 1 .
\end{aligned}
$$

This making $t_{j}$ fires and hence the next marking will be changed only for $p_{j}$ only, as $p_{i}$ is not connected with $t_{j}$

$$
\begin{aligned}
\mu_{t_{j}}^{1}\left(p_{j}\right) & =\mu^{0}\left(p_{j}\right)-I^{-}\left(p_{j}, t_{j}\right)+I^{+}\left(p_{j}, t_{j}\right) \\
& =1-1+0=0 .
\end{aligned}
$$

Thus,

$$
\mu_{t_{j}}^{1}=(1,1, \ldots, 1,0, \ldots, 1)
$$

As, we have considered PN is a Boolean Petri net, i.e., it must generate all the Boolean $n$-vectors that means there exists at least one firing sequence which generates the marking vector where $j^{t h}$ marking is 1 and $i^{\text {th }}$ marking is 0 i.e., $\mu^{k}=(1,1, \ldots, 0,1, \ldots, 1)$.

With this marking, we can check that the transition $t_{j}$ will fire as it only connected with $p_{j}$, as

$$
\begin{aligned}
I^{-}\left(p_{j}, t_{j}\right) & \leq \mu^{k}\left(p_{j}\right) \\
1 & \leq 1 .
\end{aligned}
$$

After the firing of $t_{j}$ the next marking will be

$$
\begin{aligned}
\mu^{k+1}\left(p_{j}\right) & =\mu^{k}\left(p_{j}\right)-I^{-}\left(p_{j}, t_{j}\right)+I^{+}\left(p_{j}, t_{j}\right) \\
& =1-1+0=0 .
\end{aligned}
$$

Thus, $\mu^{k+1}=(0,0, \ldots, 0,0, \ldots, 0)$.

This marking will be the same when we will consider the marking $\mu_{t_{i}}^{1}=(1,1, \ldots, 0,0, \ldots, 1)$ from the subnet, thus contradicting the concept of crispness. 
International Journal of Mathematical, Engineering and Management Sciences

Vol. 5, No. 1, 83-95, 2020

https://doi.org/10.33889/IJMEMS.2020.5.1.008

\subsection{For Subnet-4}

Let us suppose $P N$ contains subnet-4 structure as an induced subnet. In this case, the cardinality of the places should be strictly greater than two i.e., $n>2$. In this case by the firing of $t_{i}$ and $t_{j}$ leads to the marking $(1,1, \ldots, 0,1, \ldots, 1)$ and $(1,1, \ldots, 1,0, \ldots, 1)$. Thus, if it is an induced subnet it will never lead to the zero vector by any firing sequence i.e., $(0,0, \ldots, 0,0, \ldots, 0)$ will never be obtained. This contradicts the Boolean property of the Petri net. While, if there exists a transition say $t_{k}$, which is only connected with $p_{i}$ and $p_{j}$ with $I^{-}\left(p_{i}, t_{k}\right)=1$ and $I^{-}\left(p_{j}, t_{k}\right)=$ 1 , then the zero vector can be obtained but in case many Boolean vectors will be repeated which contradicts the crispness of the Petri net.

Thus, we can conclude that while modeling any system if it contains these four structures as its induced subnet or a subnet induced to these forbidden subnets, then either binary $n$-vectors or the crisp binary $n$-vectors will not be generated. In other words, one can say that the subnet1 , subnet-2, subnet-3 and subnet- 4 are the forbidden structures for any 1-safe Petri net to be a Crisp Boolean Petri net.

\section{Discussion and Validation}

Analyzing forbidden structures from any family of graphs is always a good platform from a research point of view. This leads to the characterization of any particular type of graph or family of the graph. Earlier types of researches have been proposed in the area of graph theory. In (Greenwell and Hemminger, 1972) some forbidden graphs are shown from the family of graphs which has their line graphs as a Planer graph. Authors in (Hogben and Van Der Holst, 2007) shows some forbidden minor graphs for the family of graphs whose maximum co-ranks is less than or equal to 2. Similarly, in (Tondato et al., 2005) authors proposed thirteen forbidden sub-graphs for the Path graphs. For some family of graphs forbidden subgraph characterization has also be done like for Interval Graph (Lekkeikerker and Boland, 1962) for Star like Graph (Szwarcfiter and Cerioli, 1999) for Directed Path Graph (Panda, 1999) etc. Some Petri net software is freely available to validate the models represented by Petri net like PIPE v4.3.0, Snoopy, WoPeD etc. We have validated by using PIPE v.4.3.0 for all the forbidden subnets for both the cases when it contradicts for BPN or CBPN. In Figure 5 the Petri net contains subnet-1 as an induced subnet and its reachability shows that all the binary vectors are generated but are not crisp as there exists some cycle which shows the vectors $(0,0,0)$ and $(1,0,0)$ are repeating in the markings.
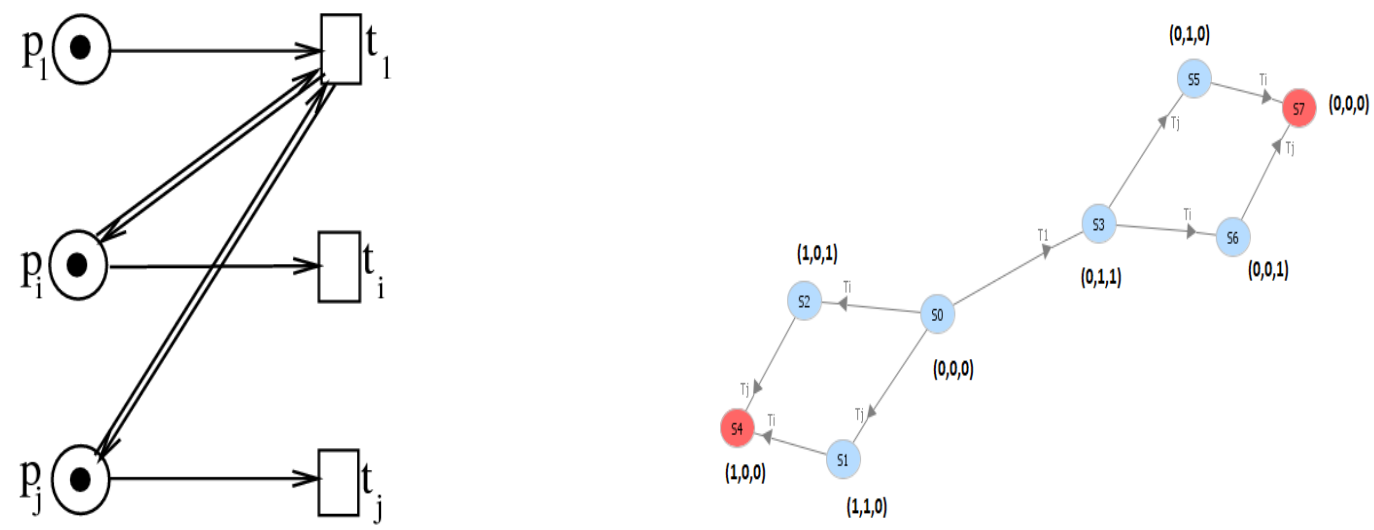

Figure 5. Petri net containing subnet-1 and its coverability graph 
International Journal of Mathematical, Engineering and Management Sciences

Vol. 5, No. 1, 83-95, 2020

https://doi.org/10.33889/IJMEMS.2020.5.1.008

Again, in Figure 6, the Petri net containing subnet-2 as an induced subnet and its reachability graph shows that all the Boolean vectors are not generated. In Figure 7, Petri net contains subnet-3 as an induced subnet and its reachability graph shows that all the Boolean vectors are not generated and in Figure 8, Petri net contains subnet-4 as an induced subnet and its reachability graph shows that all the Boolean vectors are not generated.
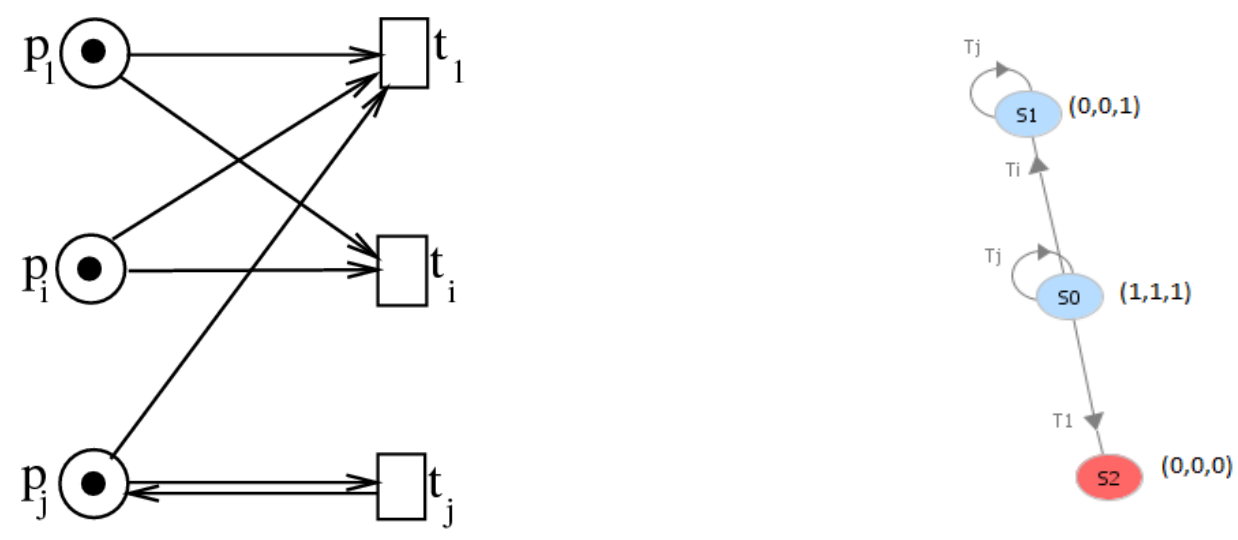

Figure 6. Petri net containing subnet-2 and its coverability graph
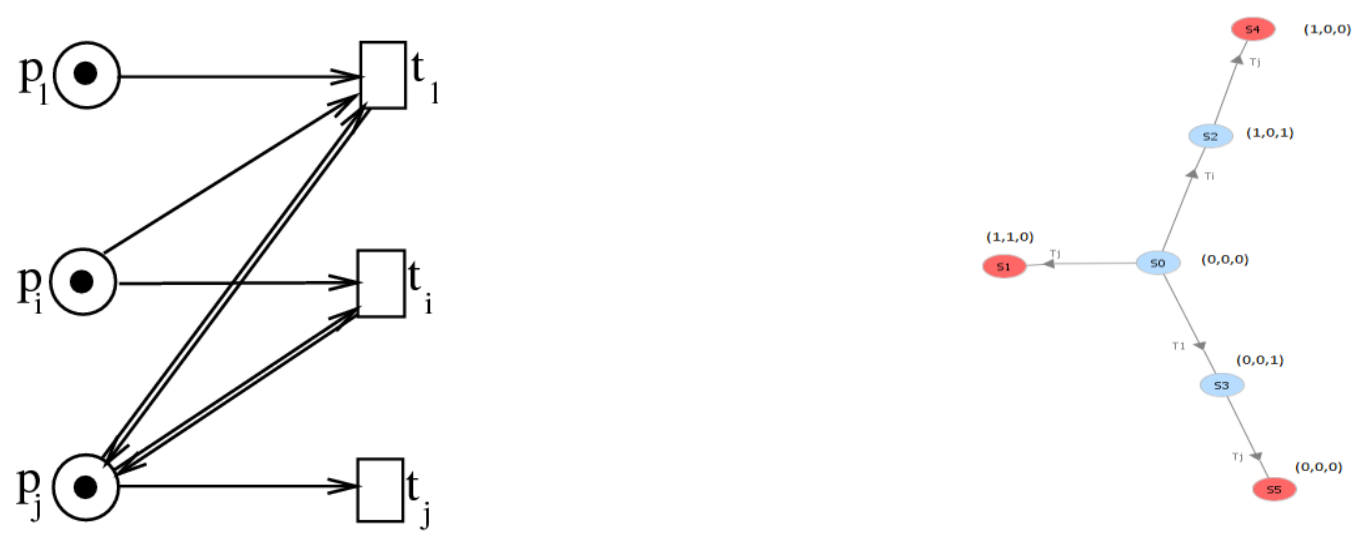

Figure 7. Petri net containing subnet-3 and its coverability graph 
International Journal of Mathematical, Engineering and Management Sciences

Vol. 5, No. 1, 83-95, 2020

https://doi.org/10.33889/IJMEMS.2020.5.1.008
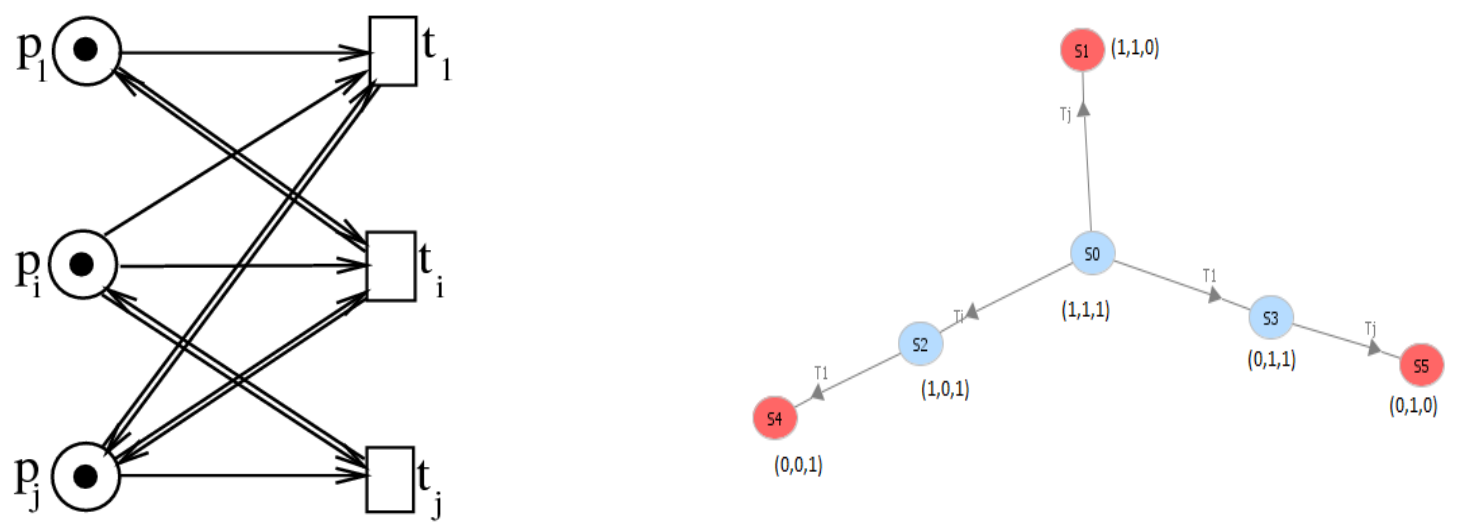

Figure 8. Petri net containing subnet-4 and its coverability graph

\section{Conclusion}

In this paper, we have considered the family of crisp Boolean Petri net graphs and proposed four forbidden subnets for any 1-safe Petri net graph. Any 1-safe Petri net behaves as CBPN if it does not contain any subnet which is homeomorphic to any of the four forbidden subnet which has shown in Figure 4. These forbidden subnets may provide a small characterization for all 1-safe Petri net to be a CBPN. Forbidden Petri nets structures can be used in the testing of algorithms for those classes of Petri nets which generates all the binary $n$-vectors. Just observing the forbidden structure one can conclude that whether the class of Petri net belongs to CBPN or not. In future, one can think about the general forbidden characterization of 1-safe Petri net to be a Crisp Boolean Petri net.

\section{Conflict of Interest}

All the authors have no conflict of interest.

\section{Acknowledgement}

The first author is thankful to the Department of Science and Technology (SERB) (PID: ECR/2017/003480) and UPOEII (ID-257) and DST purse grant for providing research facility. The authors are expressing their deep gratitude to anonymous reviewers, editors for their valuable suggestions and comments.

\section{References}

Francis, M., Hell, P., \& Stacho, J. (2015, January). Forbidden structure characterization of circular-arc graphs and a certifying recognition algorithm. In Proceedings of the Twenty-Sixth Annual ACM-SIAM Symposium on Discrete Algorithms (pp. 1708-1727). Society for Industrial and Applied Mathematics, Philadelphia, PA, USA.

Greenwell, D.L., \& Hemminger, R.L. (1972). Forbidden subgraphs for graphs with planar line graphs. Discrete Mathematics, 2(1), 31-34. 
International Journal of Mathematical, Engineering and Management Sciences

Vol. 5, No. 1, 83-95, 2020

https://doi.org/10.33889/IJMEMS.2020.5.1.008

Gupta, S., Kumawat, S., \& Singh, G.P. (2019a, July). Fuzzy Petri net representation of fuzzy production propositions of a rule based system. In International Conference on Advances in Computing and Data Sciences (pp. 197-210). Springer, Singapore.

Gupta, S., Singh, G.P., \& Kumawat, S. (2019b). Petri net recommender system to model metabolic pathway of polyhydroxyalkanoates. International Journal of Knowledge and Systems Science, 10(2), 42-59.

Harary, F. (2015). A seminar on graph theory. Courier Dover Publications.

Hogben, L., \& Van Der Holst, H. (2007). Forbidden minors for the class of graphs $\mathrm{G}$ with $\xi(G) \leqslant 2$. Linear Algebra and its Applications, 423(1), 42-52.

Jensen, K. (1993, June). An introduction to the theoretical aspects of coloured Petri nets. In Workshop/School/Symposium of the REX Project (Research and Education in Concurrent Systems) (vol. 803, pp. 230-272). Springer, Berlin, Heidelberg.

Jensen, K., \& Rozenberg, G. (2012). High-level Petri nets: theory and application. Springer Science \& Business Media.

Kansal, S., Acharya, M., \& Singh, G.P. (2011b). Uniqueness of minimal 1-safe Petri net generating all the binary $n$-vectors as its marking vectors exactly once. Scientiae Mathematicae Japonicae, $74(2 \& 3)$, 117-120.

Kansal, S., Acharya, M., \& Singh, G.P. (2012). Boolean Petri nets. In Petri Nets-Manufacturing and Computer Science, IntechOpen, 381-406.

Kansal, S., Singh, G.P., \& Acharya, M. (2010). On petri nets generating all the binary $n$-vectors. Scientiae Mathematicae Japonicae, 71(2), 209-216.

Kansal, S., Singh, G.P., \& Acharya, M. (2011a). 1-Safe Petri nets generating every binary $n$-vector exactly once. Scientiae Mathematicae Japonicae, 74(1), 29-36.

Kansal, S., Singh, G.P., \& Acharya, M. (2015). On the problem of characterizing boolean Petri nets. International Journal of Computer Applications, 128(2), 0975, 8887.

Kumar, R., Singh, G.P., Pandey, S.K., \& Shekhawat, V.J. (2018). Non-cyclic hydrocarbons: generating all the binary $n$-vectors, special issue on Mathematical Sciences (graph theory), Vigyan Garima Sindu, Commission for Scientific and Technical Terminology, Ministry of Human Resource Development, Govt. of India, ISSN: 2320-7736, 105, 24-28 (in hindi version).

Lekkeikerker, C., \& Boland, J. (1962). Representation of a finite graph by a set of intervals on the real line. Fundamenta Mathematicae, 51(1), 45-64.

Mayr, E.W. (1984). An algorithm for the general Petri net reachability problem. SIAM Journal on Computing, 13(3), 441-460.

Murata, T. (1989). Petri nets: Properties, analysis and applications. Proceedings of the IEEE, 77(4), 541-580.

Panda, B.S. (1999). The forbidden subgraph characterization of directed vertex graphs. Discrete Mathematics, 196(1-3), 239-256.

Pastor, E., Roig, O., Cortadella, J., \& Badia, R.M. (1994, May). Petri net analysis using boolean manipulation. In International Conference on Application and Theory of Petri Nets (pp. 416-435). Springer, Berlin, Heidelberg.

Petri, C.A., \& Reisig, W. (2008). Petri net. Scholarpedia, 3(4), 6477.

Piestrak, S.J. (1995). A high-speed realization of a residue to binary number system converter. IEEE Transactions on Circuits and Systems II: Analog and Digital Signal Processing, 42(10), 661-663.

Rosen, K.H., \& Krithivasan, K. (2012). Discrete mathematics and its applications: with combinatorics and graph theory. Tata McGraw-Hill Education, India. 
International Journal of Mathematical, Engineering and Management Sciences

Vol. 5, No. 1, 83-95, 2020

https://doi.org/10.33889/IJMEMS.2020.5.1.008

Singh, G.P., \& Gupta, A. (2019, March). A Petri net analysis to study the effects of diabetes on cardiovascular diseases, IEEE Xplore, ISBN: 978-93-80544-36-6 (accepted).

Singh, G.P., \& Singh, S.K. (2019). Petri net recommender system for generating of perfect binary tree. International Journal of Knowledge and Systems Science, 10(2), 1-12.

Szwarcfiter, J.L., \& Cerioli, M.R. (1999). Characterizing intersection graphs of substars of a star by forbidden subgraphs. Relatório Técnico NCE, 33(99), 1-11.

Tondato, S.B., Gutierrez, M., \& Szwarcfiter, J.L. (2005). A forbidden subgraph characterization of path graphs. Electronic Notes in Discrete Mathematics, 19, 281-287. 